\title{
A PSO-CVX Algorithm of Sum and Difference Beam Patterns for Time-Modulated Antenna Array
}

\author{
Xikuan Dong $\left(\mathbb{D}\right.$, Hailin Li $\mathbb{D}^{D}$, Jing Tan $\mathbb{D}^{D}$, Jiawen Hu $(\mathbb{D}$, and Yachao Jiang $(\mathbb{D}$ \\ College of Electronic and Information Engineering, Nanjing University of Aeronautics and Astronautics, Nanjing 210016, China \\ Correspondence should be addressed to Xikuan Dong; 1241317185@qq.com
}

Received 1 December 2020; Revised 30 January 2021; Accepted 20 February 2021; Published 3 March 2021

Academic Editor: Pierfrancesco Lombardo

Copyright (C) 2021 Xikuan Dong et al. This is an open access article distributed under the Creative Commons Attribution License, which permits unrestricted use, distribution, and reproduction in any medium, provided the original work is properly cited.

\begin{abstract}
An integrated optimization of sum and difference beam for time-modulated linear antenna array is studied in this paper. The goal of sum and difference beam synthesis is to generate sum beam in the main band and difference beams in the first-order sideband with low side-lobe level through timing switches. The turn-on times of antenna array are achieved by solving a quadratic constraint linear programming; meanwhile, the opening times are optimized by particle swarm optimization algorithm. The results of linear array show that the sum and difference beam can be scanned within \pm 40 degrees, with lower peak side-lobe level.
\end{abstract}

\section{Introduction}

Monopulse technology is a tracking method which is developed and widely used in the late 1950s. It is further adapted in a radar system, named monopulse radar [1]. Monopulse radar antenna needs to generate a main lobe sum beam and a difference beam with two main lobes. The function of the sum beam is to detect the target distance and carry out distance tracking, and the function of the difference beam is to detect the target azimuth and elevation angle information and carry out angle tracking [2]. Then, by comparing with the information obtained by the sum and difference beam, the spatial position of the target is determined [3]. If the target is exactly in the direction of the maximum value of the sum beam, the signal received by the difference beam is extremely weak. When the target moves, the signal received by the difference beam changes from weak to strong and the difference signal can be used to drive the servo mechanism to make the antenna rotate in elevation or azimuth so that the zero value direction of the difference beam is always aligned with the target track [4]. Monopulse radar has the advantages of high tracking accuracy, unlimited range, great potential for data rate, and outstanding antijamming performance. However, it can only be used for multibeam antennas, and the beam must be shaped.
In 1963, Kummer et al. first proposed a variable aperture size (Variable Aperture Sizes, VAS) time modulation method and applied it to an array antenna to achieve low/ ultralow side lobe [5]. Reyna presents a new research in the field of timed antenna arrays. The study proposes the synthesis of timed antenna array for arbitrary-shaped beam energy patterns. By using the well-known particle swarm optimization to obtain low side-lobe patterns, the results show that the timing antenna array can provide accurate beam energy patterns of any shape [6]. Reyna presents the synthesis of a conformal timed array for optimum energy patterns during beam scanning by the particle swarm optimization and simulates and verifies the semicylindrical array [7]. Garza presents the synthesis of time-domain circular antenna arrays for a flat-top energy pattern through the use of multiobjective optimization differential evolution (DEMO), comprehensive consideration of the best real-time excitation delay, and the best excitation amplitude optimization to obtain a good performance circular antenna array pattern [8]. Mahanti proposes a real-coded genetic algorithm (GA) for the optimal design of a reconfigurable dual-beam linear time-modulated array antenna that only uses phase control. The amplitude distribution will generate a pencil beam with zero phase and a flat-top beam with a continuously controllable phase analog phase shifter Alberto 
Reyna presents new designs of time-modulated antenna arrays for circularly polarized shaped beams. The time switching sequence of the elements optimized by the particle swarm algorithm is designed to design the array while maintaining a uniform amplitude. At the same time, two design examples of the circular polarization flat top and the equal-pass pattern are given [10]. Through a suitable strategy based on multiobjective particle swarm optimization, the problem of minimizing power loss in the time modulation array is solved by Poli [11].

Pal proposes a multiobjective optimization (MO) framework for designing time-modulated linear antenna arrays. The maximum side-lobe level, the main lobe beam width between the maximum sideband level, and the previous null are regarded as three simultaneous realizations. For different goals, the evolutionary algorithm using the most goals can determine the best compromise between these three goals. This provides greater flexibility in the design, and users can choose a solution that obtains an appropriate compromise as the final result [12].

In 1959, Shanks discovered the central frequency band, and the first-order sideband can be used to synthesize the sum beam and the difference beam, and it was applied to beam scanning since 1961. This is also the first time that time modulation technology has been applied to the electrical beam scanning of an array antenna without a phase shifter [13]. Tennant and his team proposed a two-element time modulation array in the United Kingdom. By adjusting the timing loaded on the two elements, the sum beam is synthesized at the center band and the difference beam is synthesized at the first-order sideband, which can be used for wide-angle targets' direction estimation [14] and experimental verification of this theory [15]. Recently, algorithms widely used in the synthesis of sum and difference beams include adaptive beamforming [16-18], evolutionary algorithms such as genetic algorithms [19], particle swarm optimization algorithms [20], and differential evolution algorithms, and their hybrid changes [21]. However, we noticed that the computational cost of the above heuristic method is higher and the convergence speed is slow. Therefore, people have begun to use convex optimization algorithms for antenna pattern synthesis. The obstacle is how to convert the nonlinear problem into a convex problem or decompose the original problem into a combination of convex and nonconvex problems. In order to solve the nonconvex optimization problem, Fan et al. first used the variant penalty to transform the original constrained optimization problem into an unconstrained nonsmooth and then used a smooth function to approximate the nonsmooth component to obtain a smooth unconstrained optimization. The problem then can be solved by the steepest descent, with precise control of different beam patterns [22]. Kwak proposed a linear programming method which simplifies the antenna system of monopulse radar by attaching a common weight (for summing beam and difference beam) to each antenna. It is computationally efficient and does not require slow global optimization techniques [23]. Dorsey proposed an A second-order cone program (SOCP) method to synthesize beams with azimuth and difference characteristics from a uniform circular array and perform electromagnetic simulation [24]. Yang Shiwen's team in China introduced the equivalent phase weighting into the time modulation technology, and the center band beam scanning and beamforming are realized without the phase shifter and attenuator [25]. Kwak proposed a new technique for finding disjoint and completely covering weight vector sets in 2019 . Each weight vector set forms a sparse subarray to form a single beam. Our algorithm decomposes the original nonconvex optimization problem into the problem of finding disjoint weight vectors [26]. Morabito proposed a new synthetic one-dimensional reconfigurable sparse array method, which can generate summation and difference power patterns. Only the phase shift is required to switch the half-array between the two radiation modes and the sparsity of the array layout can be achieved by appropriately using the principle of compressed sensing [27]. In addition to algorithmic improvements, many people have conducted research on hardware aspects such as antenna design and optimized the sum and difference beam pattern [2, 28-30].

However, nowadays, people have adopted symmetrical array element timing correlation in the research of sum and difference beams. This symmetric method needs to limit the timing switch, and there is no lower side-lobe level when the beam is scanned. The purpose of this paper is to use the PSOCVX algorithm to synthesize the sum-and-difference beam. The turn-on times of antenna array are achieved by solving a quadratic constraint linear programming; meanwhile, the opening times are optimized by particle swarm optimization algorithm, which greatly improves the time efficiency. Compared with the traditional phased array, only time modulation controls the on-off of the sequential switch and no power divider and phase shifter is required, which greatly simplifies the system complexity and reduces the cost of hardware production. And, the sum and difference beams synthesized by this algorithm also have low side-lobe when scanning within $\pm 40^{\circ}$.

The structure of this paper is arranged as follows. Section 2 introduces the basic mathematical model of equal excitation uniform time modulation linear array and the sumand-difference beam. In Section 3, the PSO-CVX algorithm will be explained in details; besides, the process of using PSO-CVS algorithm to solve the mathematical model will also be presented. In Section 4, by listing, the performance and result of sum-and-difference beam during scanning are provided to verify the effectiveness of the proposed algorithm. Finally, conclusions are drawn in Section 5.

\section{Mathematical Model}

In Figure 1, without considering the element coupling, the radiation intensity of the uniformly excited uniform linear array is [31]

$$
E(\theta, t)=e^{j 2 \pi f_{0} t} \sum_{n=1}^{N} e^{j k x_{n} \sin \theta},
$$

where $\theta$ is the radiation direction, $N$ is the number of elements, $f_{0}$ is the carrier frequency, $k=2 \pi / \lambda, \lambda$ is the 


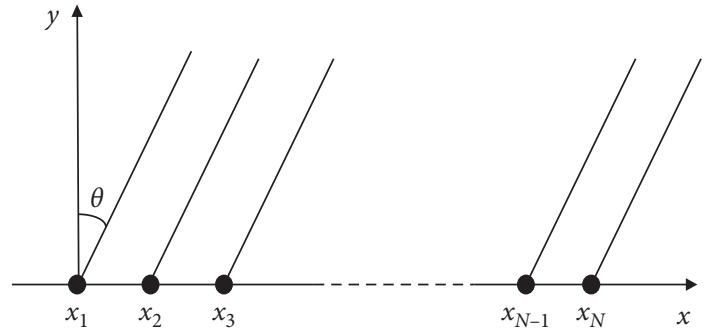

FIgURE 1: Uniform line array.

wavelength, $\lambda f_{0}=c$ is the speed of light, and $x_{n}$ is the position of the $n$ element.

Introduce the ideal rectangular pulse switch function [32] which is shown in Figure 2, and its mathematical expression is

$$
U_{n}(t)=\varepsilon\left(t-t_{n}\right)-\varepsilon\left[t-\left(t_{n}+\tau_{n}\right)\right],
$$

where $\varepsilon(t)$ is the step function, $t_{n}$ is the channel opening time, and $\tau_{n}$ is the channel keep open time. Thus, the far-field radiation intensity of the linear array based on the time modulation technology is

$$
E(\theta, t)=e^{j 2 \pi f_{0} t} \sum_{n=1}^{N} U_{n}(t) e^{j k x_{n} \sin \theta} .
$$

Fourier expansion of the rectangular pulse switching function is

$$
U_{n}(t)=\sum_{m=-\infty}^{m=+\infty} a_{m n} e^{j 2 \pi m f_{p} t}, m=0, \pm 1, \ldots, \pm \infty,
$$

where $f_{p}=1 / T_{p}, T_{p}$ is the time modulation period, and $a_{m n}$ is the $m$ th harmonic excitation.

The far-field radiation intensity of the $m$-order sideband can be obtained as

$$
E_{m}(\theta, t)=e^{j 2 \pi\left(f_{0}+m f_{p}\right) t} \sum_{n=1}^{N} a_{m n} e^{j k x_{n} \sin \theta} .
$$

The expansion coefficient of the $m$ th order sideband of the sequential switch of the $n$th element is

$$
a_{m n}=\tau_{n} f_{p} \sin c\left(\pi m \tau_{n} f_{p}\right) e^{-j \pi m f_{p}\left(2 t_{n}+\tau_{n}\right)} .
$$

Schematic diagram of sum and difference beam pattern is shown in Figure 3, and the goal of sum-and-difference beam synthesis is to form a sum beam in the main frequency band through the switching timing of the array elements, form a difference beam on the first-order sideband, and improve the performance of the sum and difference beam, that is, under normalization of the peak level of the main band. (1) The maximum side-lobe level of the main band is the smallest. (2) Side-lobe radiation on the first-order sideband is less than the constraint value $\varepsilon_{1}$. (3) The firstorder sideband produces a null in the main radiation direction, which is smaller than the constraint value $\varepsilon_{2}$. (4) The

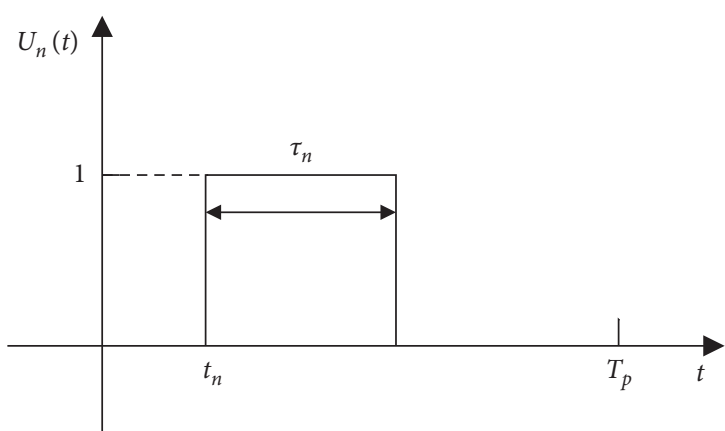

FIGURE 2: Rectangular pulse switching function.

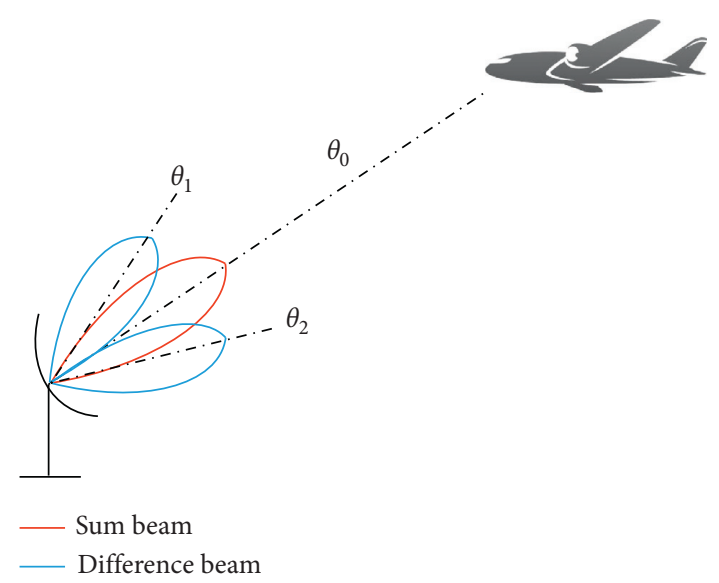

Figure 3: Sum and difference beam pattern.

first-order sideband produces a difference beam, that is, the radiation level is equal to the constraint value $\varepsilon_{3}$ in the direction of the difference beam $\theta_{1}$ and $\theta_{2}$. The mathematical model of sum and difference beam pattern synthesis is obtained by analysis as

$$
\begin{array}{cl}
\min _{t_{n}, \tau_{n}} \max & \left|\mathbf{A}_{0}^{T} \mathbf{B}_{s}\right|, \quad \theta_{s} \in \Omega_{s}, \\
& \mathbf{A}_{0}^{T} \mathbf{B}_{0}=1, \\
& \left|\mathbf{A}_{1}^{T} \mathbf{B}_{g}\right|<\varepsilon_{1}, \quad \theta_{g} \in \Omega_{g}, \\
\text { s.t., } \quad & \left|\mathbf{A}_{1}^{T} \mathbf{B}_{0}\right|<\varepsilon_{2}, \\
& \left|\mathbf{A}_{1}^{T} \mathbf{B}_{1}\right|=\varepsilon_{3}, \\
& \left|\mathbf{A}_{1}^{T} \mathbf{B}_{2}\right|=\varepsilon_{3},
\end{array}
$$

where $\mathbf{A}_{0}=\left[a_{01}, \ldots, a_{0 N}\right]^{T}$ and $\mathbf{A}_{1}=\left[a_{11}, \ldots, a_{1 N}\right]^{T}$ are the expansion coefficient vectors, $\Omega_{s}$ is main band side-lobe area, $\mathbf{B}_{s}=\left[e^{j k x_{1} \sin \theta_{s}}, \ldots, e^{j k x_{N} \sin \theta_{s}}\right]^{T}$ is the steering vector of the side-lobe sampling angle of the main band, $\Omega_{g}$ is the first-order sideband side-lobe area, $\mathbf{B}_{g}=\left[e^{j k x_{1} \sin \theta_{g}}, \ldots\right.$, $\left.e^{j k x_{N} \sin \theta_{g}}\right]^{T}$ is the steering vector of the first-order side-lobe sampling angle, $\mathbf{B}_{0}$ is the steering vector in the beam direction $\theta_{0}$, and $\mathbf{B}_{1}$ and $\mathbf{B}_{2}$ is the steering vector in the direction of the difference beam $\theta_{1}$ and $\theta_{2}$. 


\section{PSO-CVX Algorithm}

In equation (7), the optimization model optimizes the main frequency band, first-order sideband main lobe, and sidelobe levels. It is a nonlinear optimization problem. We propose an optimization algorithm based on PSO-CVX to solve this nonlinear optimization problem.

When $m=0$, from equation (6), we can get $a_{0 n}=\tau_{n} f_{p}$; thus, the electric field strength of the main frequency band is

$$
E_{0}(\theta)=\sum_{n=1}^{N} a_{0 n} e^{j k x_{n} \sin \theta}
$$

For the electric field of the main frequency band, $e^{j 2 \pi f_{0} t}$ is a common factor and can be omitted. When $x_{n}$ is known, $E_{0}(\theta)$ is a linear function of $a_{0 n}$. The main frequency band and beamforming problem is a subproblem of the optimization problem of formula (7), which can be expressed as

$$
\begin{aligned}
& \min _{a_{0 n}}, \max \left|\mathbf{A}_{0}^{T} \mathbf{B}_{s}\right|, \quad \theta_{s} \in \Omega_{s} \\
& \text { s.t., } \quad \mathbf{A}_{0}^{T} \mathbf{B}_{0}=1 .
\end{aligned}
$$

The global optimal solution of $a_{0 n}$ can be obtained by convex optimization algorithm. Introducing the slack variable with side-lobe level constraint $\varepsilon$, convert the minimummaximization problem of formula (9) into an inequality constrained optimization problem:

$$
\begin{array}{ll}
\min _{a_{0 n}} \varepsilon & \\
& \mathbf{A}_{0}^{T} \mathbf{B}_{0}=1, \\
\text { s.t. } & \left|\mathbf{A}_{0}^{T} \mathbf{B}_{s}\right| \leq \mathcal{E}, \quad s=1, \ldots, S, \\
& -\varepsilon \leq 0 .
\end{array}
$$

Let the variables in $R^{N}$ space $\mathbf{f}=\left[\begin{array}{c}1 \\ 0_{N} \\ 0_{N}\end{array}\right], \mathbf{y}=\left[\begin{array}{c}\mathcal{\varepsilon} \\ \operatorname{Re}\left(\mathbf{A}_{0}\right) \\ \operatorname{Im}\left(\mathbf{A}_{0}\right)\end{array}\right]$, $\mathbf{c}_{0}=\left[\begin{array}{c}0 \\ \operatorname{Re}\left(\mathbf{B}_{0}\right) \\ \operatorname{Im}\left(\mathbf{B}_{0}\right)\end{array}\right], \quad \mathbf{d}_{0}=\left[\begin{array}{c}0 \\ \operatorname{Im}\left(\mathbf{B}_{0}\right) \\ -\operatorname{Re}\left(\mathbf{B}_{0}\right)\end{array}\right], \quad \mathbf{c}_{s}=\left[\begin{array}{c}0 \\ \operatorname{Re}\left(\mathbf{B}_{s}\right) \\ \operatorname{Im}\left(\mathbf{B}_{s}\right)\end{array}\right], \quad$ and $\mathbf{d}_{s}=\left[\begin{array}{c}0 \\ \operatorname{Im}\left(\mathbf{B}_{s}\right) \\ -\operatorname{Re}\left(\mathbf{B}_{s}\right)\end{array}\right]$; thus, equation (10) is transformed into a standard form of Quadratic Constraint Linear Programming (QCLP):

$$
\begin{array}{ll}
\min _{y} & \mathbf{f}^{T} \mathbf{y} \\
& \mathbf{c}_{0}^{T} \mathbf{y}=1, \\
& \mathbf{d}_{0}^{T} \mathbf{y}=0, \\
\text { s.t. } & \left(\mathbf{c}_{s}^{T} \mathbf{y}\right)^{2}+\left(\mathbf{d}_{s}^{T} \mathbf{y}\right)^{2}-\mathbf{f}^{T} \mathbf{y} \leq 0, \\
& -\mathbf{f}^{T} \mathbf{y} \leq 0 .
\end{array}
$$

The CVX toolbox can be used to obtain the global optimal solution of $\mathbf{y}$, thereby obtaining the global optimal solution of $a_{0 n}$.
When $m=1$, from equation (6), $a_{1 n}=\tau_{n} f_{p} \sin c\left(\pi \tau_{n} f_{p}\right)$ $e^{-j \pi f_{p}\left(2 t_{n}+\tau_{n}\right)}=c_{n} e^{-j 2 \pi f_{p} t_{n}}$ When $a_{0 n}$ is known, it is a certain value, so the first-order sideband electric field intensity is

$$
E_{1}(\theta)=\sum_{n=1}^{N} c_{n} e^{j 2 \pi\left(\left(x_{n} / \lambda\right) \sin \theta-f_{p} t_{n}\right)} .
$$

For the first sideband electric field, $e^{j 2 \pi\left(f_{0}+f_{p}\right) t}$ is a common factor and can be omitted. $E_{1}(\theta)$ is a nonlinear function with respect to $t_{n}$. So, the first-order sideband difference beamforming problem can be converted into a single-objective optimization problem, as shown in Figure 4.

It is required that the sum of errors between the formation of the pattern and the given pattern is the smallest and expressed as

$$
\begin{aligned}
& \min _{t_{n}}\left(\left|\mathbf{A}_{1}^{T} \mathbf{B}_{0}\right|-\varepsilon_{2}\right)+|| \mathbf{A}_{1}^{T} \mathbf{B}_{1}\left|-\varepsilon_{3}\right|+|| \mathbf{A}_{1}^{T} \mathbf{B}_{2}\left|-\varepsilon_{3}\right| \\
& \quad+\left(\max \left|\mathbf{A}_{1}^{T} \mathbf{B}_{g}\right|-\varepsilon_{1}\right), \quad \theta_{g} \in \Omega_{g} .
\end{aligned}
$$

We use particle swarm algorithm to find the best opening moment $t_{n}$ and use $N$-dimensional particles to represent $t_{n}$. The fitness function of particle swarm optimization is

$$
\begin{aligned}
\operatorname{fit}\left(t_{n}\right)= & \left(\left|\mathbf{A}_{1}^{T} \mathbf{B}_{0}\right|-\varepsilon_{2}\right)+|| \mathbf{A}_{1}^{T} \mathbf{B}_{1}\left|-\varepsilon_{3}\right|+|| \mathbf{A}_{1}^{T} \mathbf{B}_{2}\left|-\varepsilon_{3}\right| \\
& +\left(\max \left|\mathbf{A}_{1}^{T} \mathbf{B}_{g}\right|-\varepsilon_{1}\right) .
\end{aligned}
$$

The optimization goal is to minimize fit $\left(t_{n}\right)$.

In each iteration, the particle velocity and position update formula of the PSO algorithm is

$$
\begin{aligned}
v_{i d}^{t} & =\mathrm{CFa} \times\left[v_{i d}^{t-1}+\varphi_{1} r_{1}\left(\text { pbest }_{i d}-x_{i d}^{t-1}\right)+\varphi_{2} r_{2}\left(\text { gbest }_{i d}-x_{i d}^{t-1}\right)\right], \\
x_{i d}^{t} & =x_{i d}^{t-1}+v_{i d}^{t-1},
\end{aligned}
$$

where $v_{i d}^{t}$ and $x_{i d}^{t}$ are the velocity and position of the $d$ dimension of the particle $i$ in the $t$ th iteration, shrinkage factor $\mathrm{CFa}=2 /\left|2-\varphi-\sqrt{\varphi^{2}-4 \varphi}\right|, \varphi=\varphi_{1}+\varphi_{2}, \quad \varphi_{1}$ and $\varphi_{2} \quad$ are learning factors, pbest $_{i d}$ is the historical optimal value for each particle, gbest $_{i d}$ is the particle global optimal value, and $r_{1}$ and $r_{2}$ is a random number of $[0,1]$.

The processing flow of the PSO-CVX algorithm is given below, and the flowchart of the PSO-CVX algorithm is shown in Figure 5:

(1) Give the number of array elements, element position, beam width, difference beam shape, and angular sampling interval.

(2) Initialize the dimensions of the particle swarm and the initial velocity of the particles, and give the maximum number of iterations.

(3) Calculate steering vector.

(4) Calculate the optimal opening time of each array element through the convex optimization algorithm.

(5) Use PSO algorithm to optimize switch opening time: 


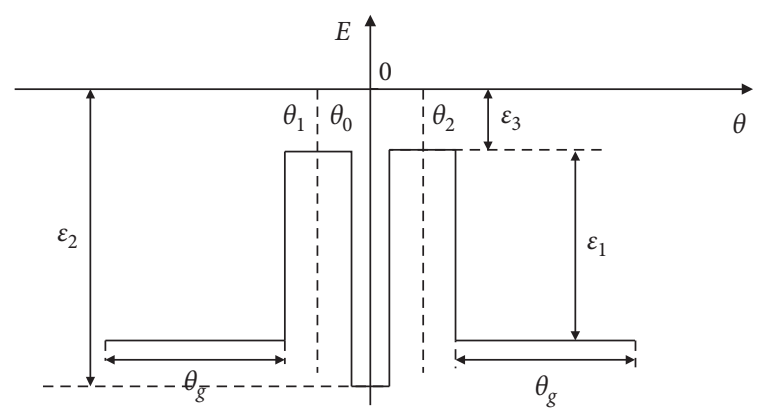

Figure 4: Constraint pattern.

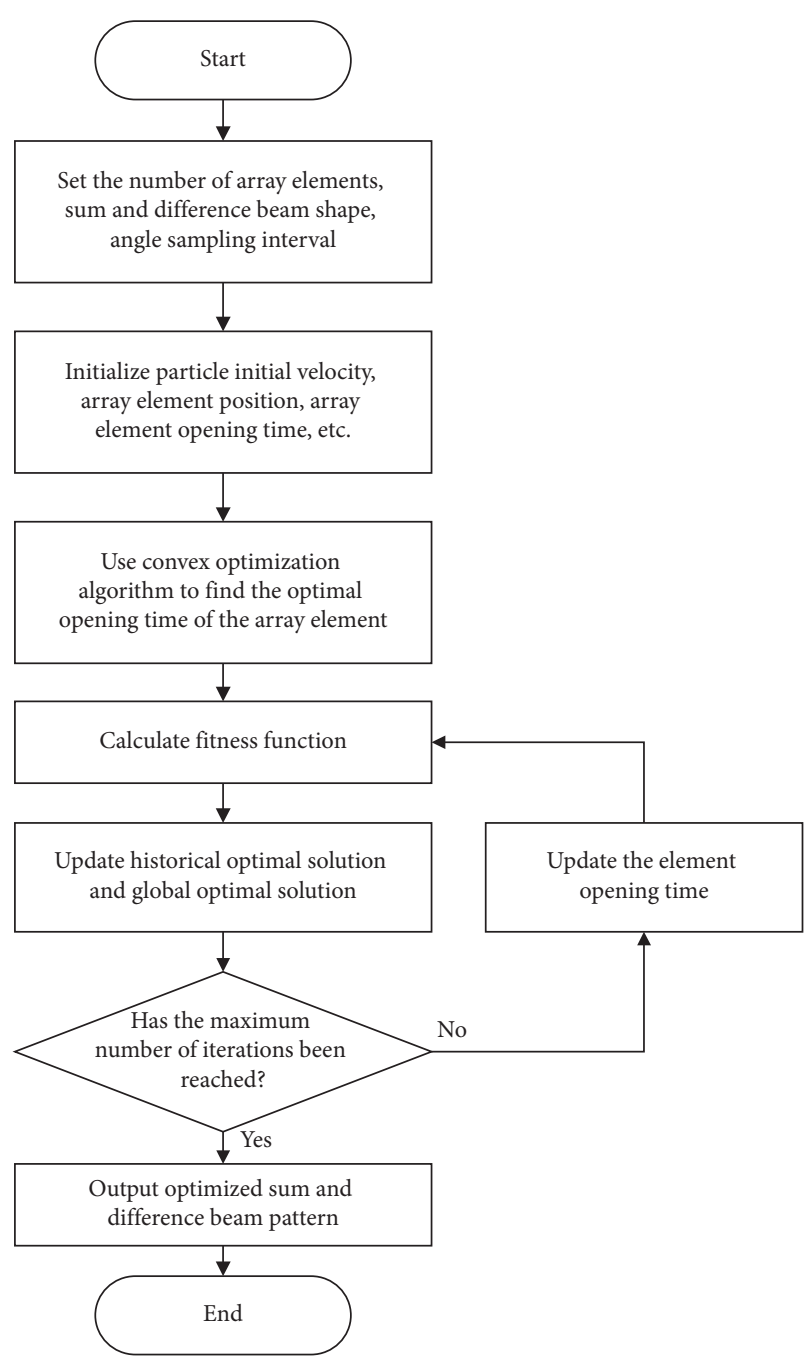

FIgURE 5: PSO-CVX flowchart.

(a) Calculate the fitness function value.

(b) Update the global optimal solution and historical optimal solution at the moment of opening.

(c) If the maximum number of iterations is reached, execute (5), otherwise execute (e).

(d) The iteration counter is incremented by 1 .

(e) Update the opening time and execute (a).

(6) End the iteration and get the optimized sum and difference beam pattern.

\section{Simulation Results}

4.1. Performance Analysis of Sum and Difference Beam Pattern. In order to verify the correctness and effectiveness of the PSO-CVX algorithm optimization and the difference beam pattern, simulations are carried out and compared with the literature $[18,20,33]$. The basic parameters of the array are shown in Table 1 . The constraint value is set to $\varepsilon_{1}=-15 \mathrm{~dB}, \varepsilon_{2}=-30 \mathrm{~dB}$, and $\varepsilon_{3}=-6 \mathrm{~dB}$.

The PSO algorithm parameter settings are shown in Table 2.

The integrated sum and difference beam pattern is shown in Figure 6. The optimized sum beam width is $20^{\circ}$, difference beam width is $16^{\circ}$, the peak side-lobe level of the main band is $-30 \mathrm{~dB}$, and the first-order sideband side-lobe peak level is $-21 \mathrm{~dB}$.

Table 3 shows the comparison of the pattern parameters of this article and the literature $[18,20,33]$.

Comparing with literature [18], it can be seen that when the peak level of the side lobe is the same as that of the beam side lobe, the peak of the side lobe of the difference beam is reduced, and the beam width is obviously narrowed to $16^{\circ}$. Compared with literature [20], the peak side-lobe of the difference beam is $1.5 \mathrm{~dB}$ higher, but the difference beam width is reduced by $2.4^{\circ}$. However, the peak side-lobe of the sum beam is significantly reduced by $10 \mathrm{~dB}$, and the sum beam width is narrowed. Compared with literature [33], when the beam width is the same as that of the beam, the peak side-lobe of the beam is reduced by $10 \mathrm{~dB}$, the peak of the side-lobe of the difference beam is reduced, and the beam width is reduced by $2^{\circ}$.

Literature $[18,20,33]$ uses the method of symmetric array element timing delay $T_{P} / 2$ to reduce the dimension. In this paper, the convex optimization algorithm is introduced to directly obtain the opening time of each array element, which reduces the dimensionality of the optimization problem and does not require conditions related to the timing of symmetric array elements. The optimized timing switch is shown in Figure 7. The specific data of optimized timing switch are shown in Table 4.

In this paper, based on the convex optimization algorithm, the global optimal solution of the turn-on time of the array element timing switch is obtained under the condition of the beam constraint, only the PSO algorithm is used to solve the turn-on time, which reduces the computational complexity and the number of iterations is significantly reduced, as shown in Figure 8, and converged in 58 iterations. The literature [20] only converged after 2000 iterations, and the literature [33] only converged after 820 iterations.

\subsection{Performance Analysis of Sum and Difference Beam Angle} Scanning. Literature $[18,33]$ can only generate sum and difference beams in a fixed direction and cannot perform angle scanning. The beam scanning experiment was carried out in literature [20]. Through the algorithm in literature [20], the peak level of the main band is $-21.6 \mathrm{~dB}$ and the peak level of the first-order sideband is $-20 \mathrm{~dB}$ when the beam is 
TABLE 1: Linear array parameter initialization.

\begin{tabular}{lc}
\hline Parameter name & Parameter value \\
\hline Carrier frequency $\left(f_{0}\right)$ & $5 \times 10^{9} \mathrm{~Hz}$ \\
Main radiation direction $\left(\theta_{M}\right)$ & $0^{\circ}$ \\
Array element spacing & $\lambda / 2$ \\
Number of array elements & 16 \\
Sampling rate & $1^{\circ}$ \\
Difference beam direction $\left(\theta_{1,2}\right)$ & $\pm 7^{\circ}$ \\
\hline
\end{tabular}

TABLE 2: PSO algorithm parameter initialization.

\begin{tabular}{lc}
\hline Parameter name & Parameter value \\
\hline Population size & 200 \\
Weight coefficient & 0.73 \\
Particle swimming range & {$[0,1]$} \\
Particle velocity range & {$[-0.1,0.1]$} \\
Time modulation frequency & $40 \mathrm{Mhz}$ \\
The maximum number of iterations & 200 \\
Learning factor & $\varphi_{1}=\varphi_{2}=2.05$ \\
\hline
\end{tabular}

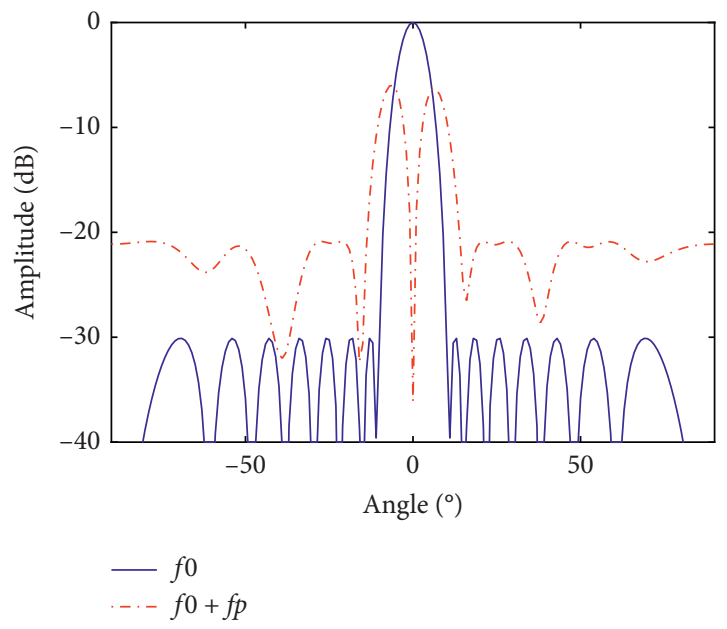

Figure 6: Sum and difference beam pattern $\left(\theta_{M}=0\right)$.

TABLE 3: Comparison of sum and difference beam parameters.

\begin{tabular}{lcccc}
\hline Optimization & $\begin{array}{c}\text { Literature } \\
{[18]}\end{array}$ & $\begin{array}{c}\text { Literature } \\
{[20]}\end{array}$ & $\begin{array}{c}\text { Literature } \\
{[33]}\end{array}$ & $\begin{array}{c}\text { PSO- } \\
\text { CVX }\end{array}$ \\
\hline$\varepsilon(\mathrm{dB})$ & -30 & -19.8 & -20 & -30.1 \\
$\varepsilon_{3}(\mathrm{~dB})$ & -6.1 & -6 & $/$ & -6 \\
$\varepsilon_{1}(\mathrm{~dB})$ & -14.6 & -16.5 & -14.8 & -15 \\
$\mathrm{BW0}^{\circ}$ & 21.4 & 21.6 & 20 & 20 \\
$\mathrm{BW}^{\circ}$ & 28.8 & 18.4 & 18 & 16 \\
\hline
\end{tabular}

pointing at $\pm 30^{\circ}$. In order to verify the radiation performance of the algorithm in this paper during beam scanning, the following experiments are carried out by changing the beam direction.

Let $\theta_{M}=10,20,30,40$. When the beam is scanned, the performance of the sum and difference beams decreases, and the constraint value $\varepsilon_{1}$ and $\varepsilon_{2}$ is relaxed. Let $\varepsilon_{1}=-10$ and $\varepsilon_{2}=-25$ perform simulation to get the $\theta_{M}$ changed sum and

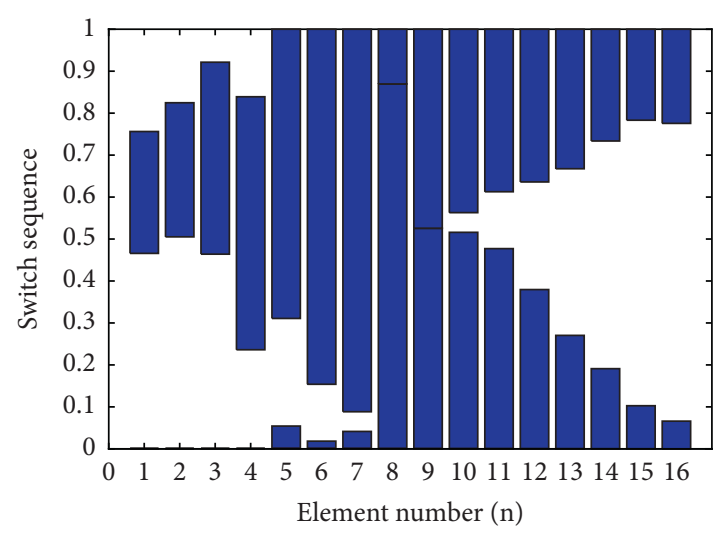

Figure 7: Time series distribution diagram.

Table 4: Timing stimulus table.

\begin{tabular}{lcc}
\hline Element no. & Turn-on time $t_{n}$ & Opening time $\tau_{n}$ \\
\hline 1 & 0.4658 & 0.2904 \\
2 & 0.5052 & 0.3196 \\
3 & 0.4640 & 0.4574 \\
4 & 0.2362 & 0.6029 \\
5 & 0.3108 & 0.7435 \\
6 & 0.1539 & 0.8644 \\
7 & 0.0884 & 0.9531 \\
8 & 0.8696 & 1.0000 \\
9 & 0.5258 & 1.0000 \\
10 & 0.5629 & 0.9531 \\
11 & 0.6127 & 0.8644 \\
12 & 0.6360 & 0.7435 \\
13 & 0.6675 & 0.6029 \\
14 & 0.7336 & 0.4574 \\
15 & 0.7832 & 0.3196 \\
16 & 0.7756 & 0.2904 \\
\hline
\end{tabular}

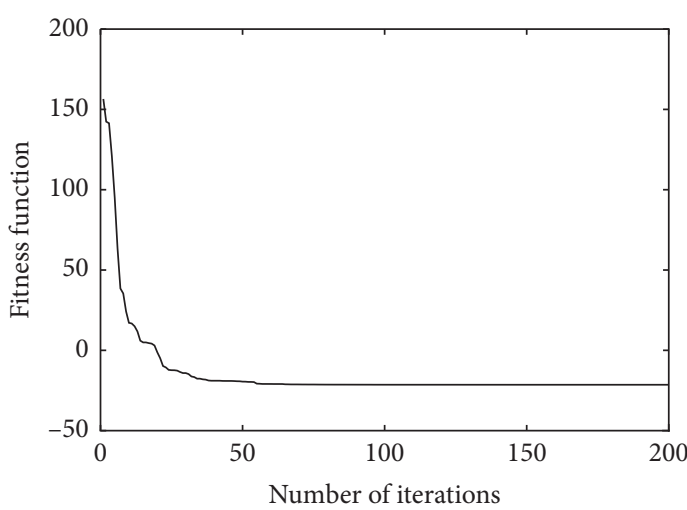

FIGURE 8: Fitness function convergence curve.

difference beam as shown in Figure 9. The sum and difference beam synthesized by the algorithm in this paper can be scanned. As $\theta_{M}$ increases, the peak level of the main frequency band and beam side-lobe gradually increases and the peak level of the first-order sideband difference beam side-lobe and null value also gradually increases. The specific parameters are given in Table 5. Compared with the literature [20], when the first-order sideband peak level is 

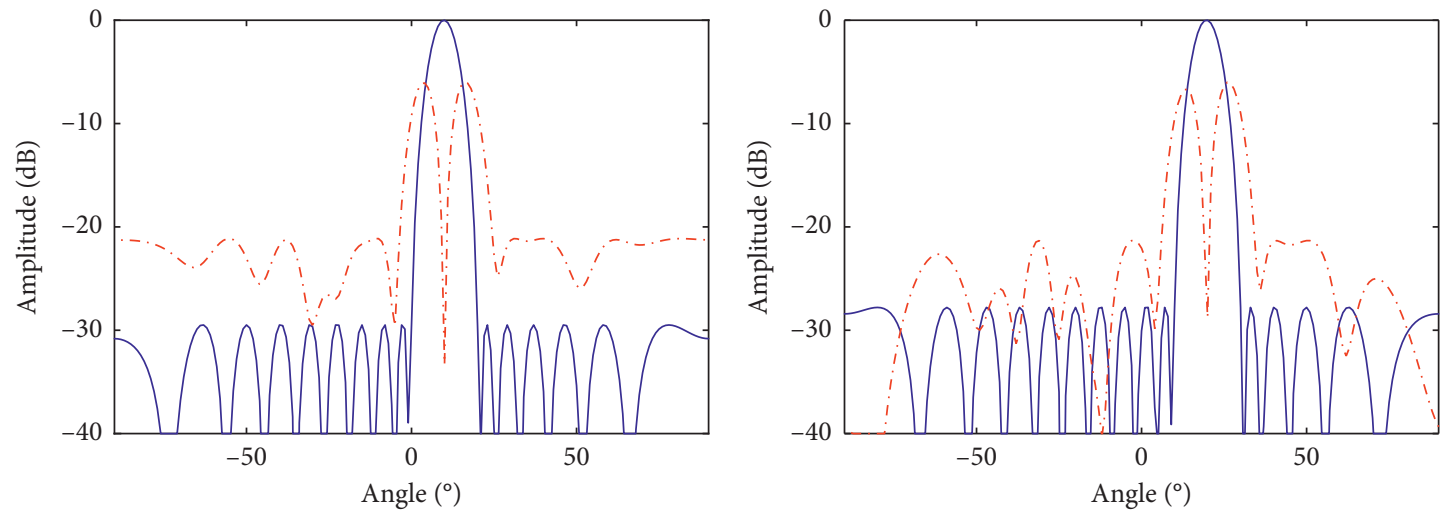

$-f 0$
$-\cdot-f 0+f p$

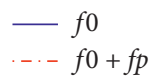

(a)

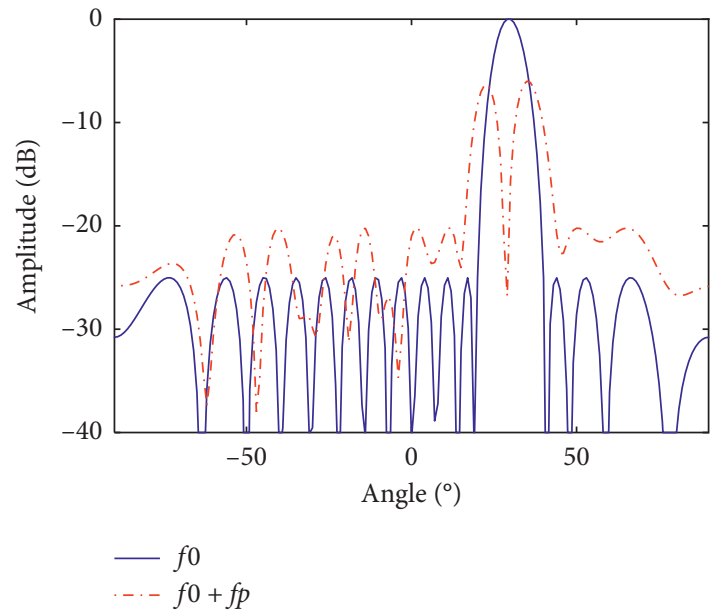

(c)

(d)

FigURE 9: Sum and difference beam under angle scan: (a) $\theta_{M}=10$, (b) $\theta_{M}=20$, (c) $\theta_{M}=30$, and (d) $\theta_{M}=40$.

TABLE 5: Sum and difference beam parameter comparison when $\theta_{M}$ changes.

\begin{tabular}{lcccc}
\hline$\theta_{M}{ }^{\circ}$ & 10 & 20 & 30 & 40 \\
\hline$\varepsilon(\mathrm{dB})$ & -29 & -27.8 & -25 & -21.5 \\
$\varepsilon_{1}(\mathrm{~dB})$ & -15.4 & -14.7 & -14.1 & -13 \\
$\varepsilon_{2}(\mathrm{~dB})$ & -33 & -28.7 & -26.8 & -26 \\
\hline
\end{tabular}

basically the same, the main frequency band peak level decreases by $3.4 \mathrm{~dB}$ at $30^{\circ}$.

During change, $\theta_{M}=50$, the simulation diagram of the sum and difference beam is shown in Figure 10. At this time, the beam side-lobe peak is $-17 \mathrm{~dB}$ and the first-order sideband side-lobe peak level is $-21 \mathrm{~dB}$, which cannot produce a null in the radiation direction to form a difference beam.

It can be seen from the simulation results that the sum and difference beams synthesized by the local algorithm have a good effect when scanning within $\pm 40^{\circ}$.

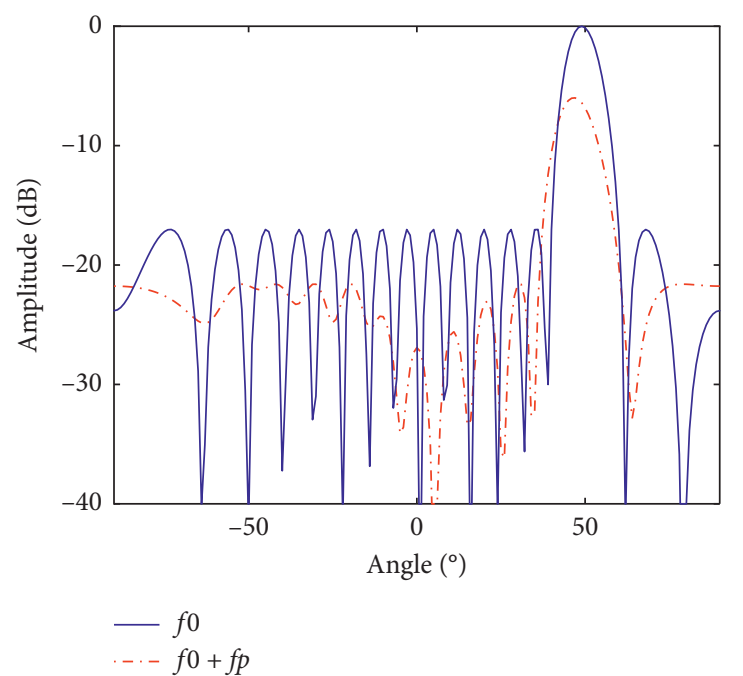

FIGURE 10: Sum and difference beam under angle scan $\left(\theta_{M}=50\right)$. 


\section{Conclusion}

This paper proposed a PSO-CVX algorithm to synthesize the sum and difference beams and to scan in $\pm 40^{\circ}$. Our basic idea is to combine the main band synthesis and beam with the convex optimization algorithm to optimize the optimal turn-on time of each element, synthesize the difference beam in the first-order sideband, and optimize the optimal opening time of each element through the PSO algorithm. The time-only modulation used in this article does not require phase shifters and power dividers, which extremely reduces the system complexity. As an example, this paper simulates a 16-element linear array. The simulation results show the effectiveness of the algorithm. Compared with other algorithms, the algorithm in this paper greatly improves the time efficiency. Compared with the reference, when the main lobe width remains the same, the peak side-lobe level of the main band drops by $10 \mathrm{~dB}$, and while the width of the main lobe of the first-order sideband is narrowed, the peak side-lobe level is reduced by $0.4 \mathrm{~dB}$. Meanwhile, it does not require the timing-related properties of symmetrical elements. Last but not the least, it can still maintain good beam performance when scanning at a larger angle. We will further optimize our model and expand the algorithm into the area array and circular array.

\section{Data Availability}

The data used to support the findings of this study are available from the corresponding author upon request.

\section{Conflicts of Interest}

The authors declare that there are no conflicts of interest regarding the publication of this paper.

\section{Acknowledgments}

This work was supported by the National Natural Science Foundation of China (Grant no. 61671239) and Fundamental Research Funds for the Central Universities (Grant nos. Kfjj20200417 and Kfjj20200412).

\section{References}

[1] Z. Wang, Y. Zeng, M. Liu et al., "Investigation of the related factors of angle measurements precision in monopulse radar," in Proceedings of the 2017 International Applied Computational Electromagnetics Society Symposium (ACES), IEEE, Suzhou, China, August 2017.

[2] Y. Chen and R. G. Vaughan, "Sum and difference beam patterns in resonant slotted-waveguide array," in Proceedings of the 2017 XXXIInd General Assembly and Scientific Symposium of the International Union of Radio Science (URSI GASS), IEEE, Montreal, Canada, August 2017.

[3] S. T. Louise, B. Allen, C. G. Parini et al., "Monopulse sum and difference signals with compensation for a failed feed element," Iet Microwaves Antennas and Propagation, vol. 10, no. 6, pp. 645-650, 2016.
[4] Y. Xiao-Qian, L. Hai-Bo, J. Han, and S. Mengmeng, "The study of mono-pulse angle measurement based on digital array radar," in Proceedings of the IET International Radar Conference, pp. 1-5, Xi'an, China, 2013.

[5] W. Kummer, A. Villeneuve, T. Fong et al., "Ultra-low sidelobes from time-modulated arrays," IEEE, vol. 11, no. 6, pp. 0-639, 1963.

[6] A. R. Maldonado, M. A. Panduro, and C. D. Rio, "Synthesis of timed antenna arrays for arbitrary shaped-beam energy patterns," in Proceedings of the 2015 9th European Conference on Antennas and Propagation (EuCAP), IEEE, Lisbon, Portugal., April 2015.

[7] A. R. Maldonado, M. A. Panduro, and C. D. R. Bocio, "Conformal timed antenna array for optimum scanned energy patterns," in Proceedings of The 10th European Conference on Antennas and Propagation, IEEE, Davos, Switzerland, April 2016.

[8] L. A. Garza, M. A. Panduro, and D. H. Covarrubias, "Timedomain synthesis of UWB circular antenna array for flat-top patterns," in Proceedings of the Loughborough Antennas \& Propagation Conference, IEEE, Loughborough, UK, November 2016.

[9] G. K. Mahanti, S. Das, and A. Chakraborty, "Design of phasedifferentiated reconfigurable array antennas with minimum dynamic range ratio," IEEE Antennas and Wireless Propagation Letters, vol. 5, pp. 262-264, 2006.

[10] A. Reyna, L. I. Balderas, and M. A. Panduro, "Time-modulated antenna arrays for circularly polarized shaped beam patterns," IEEE Antennas and Wireless Propagation Letters, p. 1, 2017.

[11] L. Poli, P. Rocca, L. Manica, and A. Massa, "Handling sideband radiations in time-modulated arrays through particle swarm optimization," IEEE Transactions on Antennas and Propagation, vol. 58, no. 4, pp. 1408-1411, 2010.

[12] S. Pal, S. Das, and A. Basak, "Design of time-modulated linear arrays with a multi-objective optimization approach," Progress In Electromagnetics Research B, vol. 23, no. 23, pp. 83-107, 2010.

[13] H. Shanks, "A new technique for electronic scanning," IRE Transactions on Antennas and Propagation, vol. 9, no. 2, pp. 162-166, 1961.

[14] A. Tennant and B. Chambers, "A two-element time-modulated array with direction-finding properties," IEEE Antennas and Wireless Propagation Letters, vol. 6, pp. 64-65, 2007.

[15] A. Tennant, "Experimental two-element time-modulated direction finding array," IEEE Transactions on Antennas and Propagation, vol. 58, no. 3, pp. 986-988, 2010.

[16] L. Zhu, S. Qiu, and Y. Han, "Combined constrained adaptive sum and difference beamforming in monopulse angle estimation," IEEE Antennas and Wireless Propagation Letters, 2018.

[17] D. L. Yan, L. R. Feng, and R. Can, "Constained adaptive monopulse algorithm based on sub-array," in Proceedings of the International Radar Conference, IET, Xian, China, April 2013.

[18] A. Tennant and B. Chambers, "Control of the harmonic radiation patterns of time-modulated antenna arrays," in Proceedings of the 2008 IEEE Antennas and Propagation Society International Symposium, pp. 1-4, San Francisco, CA, USA, July 2008.

[19] H. Cao, Z. Chen, L. Tao et al., "Synthesis of patterns for monopulse antennas with steering invariant constant beamwidth by genetic algorithm," in Proceedings of the 2016 IEEE International Symposium on Antennas and Propagation 
and USNC/URSI National Radio Science Meeting, IEEE, Puerto Rico, PR, USA, July 2016.

[20] L. Poli, P. Rocca, G. Oliveri, and A. Massa, "Harmonic beamforming in time-modulated linear arrays," IEEE Transactions on Antennas and Propagation, vol. 59, no. 7, pp. 2538-2545, 2011.

[21] J. Yang, W.-T. Li, X.-W. Shi, L. Xin, and J.-F. Yu, "A hybrid $\mathrm{ABC}-\mathrm{DE}$ algorithm and its application for time-modulated arrays pattern synthesis," IEEE Transactions on Antennas and Propagation, vol. 61, no. 11, pp. 5485-5495, 2013.

[22] X. Fan, J. Liang, X. Zhao et al., "Optimal synthesis of sum and difference beam patterns with a common weight vector for symmetric and asymmetric antenna arrays," IEEE Transactions on Antennas and Propagation, no. 99, p. 1, 2020.

[23] S. Kwak, J. Chun, D. Park, Y. K. Ko, and B. L. Cho, "Asymmetric sum and difference beam pattern synthesis with a common weight vector," IEEE Antennas and Wireless Propagation Letters, vol. 15, pp. 1622-1625, 2016.

[24] W. M. Dorsey and J. O. Coleman, "SOCP design of monopulse sum and difference beams for a uniform circular array," pp. 1-4, 2017.

[25] C. Sun, Basic Research on the Application of Space-Time FourDimensional Antenna Array in the Field of Wireless Communication, University of Electronic Science and Technology of China, Chengdu, China, 2019.

[26] S. Kwak, J. Chun, S. H. Ye et al., "Monopulse beam synthesis using a sparse single layer of weights," IEEE Transactions on Antennas and Propagation, https://arxiv.org/abs/1811.09508, 2019.

[27] A. F. Morabito and P. Rocca, "Reducing the number of elements in phase-only reconfigurable arrays generating sum and difference patterns," IEEE Antennas and Wireless Propagation Letters, vol. 14, pp. 1338-1341, 2015.

[28] S. A. Malakooti and C. Fumeaux, "Bandwidth enhancement of a double-element vivaldi antenna with sum and difference radiation patterns," in Proceedings of the 2018 Asia-Pacific Microwave Conference (APMC), Kyoto, Japan, November 2018.

[29] J. Yang, S.-S. Qi, W. Wu, and Da-G. Fang, "A novel high-gain sum and difference conical beam-scanning reflector antenna," IEEE Access, vol. 8, pp. 103291-103300, 2020.

[30] J. Guo, S. Yang, and Y. Chen, "Efficient sideband suppression in $4 \mathrm{D}$ antenna arrays with multiple time modulation frequencies," in Proceedings of the 2016 IEEE International Symposium on Antennas and Propagation and USNC/URSI National Radio Science Meeting, IEEE, Puerto Rico, PR, USA, July 2016.

[31] F. Yang, S. Yang, Y. Chen et al., "Efficient pencil beam synthesis in $4 \mathrm{D}$ antenna arrays using an iterative convex optimization algorithm," IEEE Transactions on Antennas and Propagation, no. 99, p. 1, 2019.

[32] M. A. Hannan, L. Poli, P. Rocca, and A. Massa, "Pulse sequence optimization in time-modulated arrays for secure communications," in Proceedings of the IEEE International Symposium on Antennas and Propagation, IEEE, Puerto Rico, PR, USA, July 2016.

[33] A. Mukherjee, S. K. Mandal, G. K. Mahanti et al., "Synthesis of simultaneous sum and difference patterns in uniformly excited time-modulated linear arrays using firefly algorithm," in Proceedings of the 2014 IEEE Students' Technology Symposium (TechSym), IEEE, Kharagpur, West Bengal, March 2014. 